\title{
Interfacial Shear Stresses Calculation of RC Beams Strengthened with FRP Plate under Mid-span Concentrated Loads
}

\author{
Jia Yang ${ }^{12, \text { a }}$ \\ ${ }^{1}$ College of Architectural and Civil Engineering, Shenyang University, Shenyang, China \\ ${ }^{2}$ The Key Laboratory of GeoenvironMental Engineering, Liaoning, China \\ ayangjia0918@163.com
}

Keywords: Interfacial shear stresses; FRP plate; Reinforced concrete

Abstract. Interfacial debonding failure may occur at the plate ends due to interfacial stresses. So this study analyzes the interfacial shear stresses of RC beams strengthened with FRP plate under mid-span concentrated loads. The analytical model is established, and the differential equation of interfacial shear stresses of RC beams strengthened with FRP plate is established. The calculated formula of interfacial shear stresses is deduced under mid-span concentrated loads.

\section{Introduction}

The retrofitting and strengthening of reinforced concrete structures with FRP in the civil construction panorama have become a popular technique. The use of the FRP to strengthen the concrete is an effective solution to increase the overall strength of the concrete structures. The knowledge of the precise development of the bond stresses within the interface could be very important in the design of RC beams strengthened with FRP plate. In this strengthening method, the performance of the FRP-concrete interface in providing an effective stress transfer is of crucial importance. Previous studies have shown that interfacial debonding failure will occur at the plate ends due to interfacial stresses. The mechanical properties and failure mechanism of strengthened member are closely related to bond performance of FRP-concrete interface ${ }^{[1-9]}$. So the research about the interfacial shear stresses of the FRP-concrete interface is necessary. Under external loading, the relative slip occurred on the interface between FRP plate and concrete causes the redistribution of stress and then influences mechanical behavior of component. Therefore, this paper studies on the shear stresses of FRP-concrete interface. Based on the analytical model of the interface between FRP and concrete, the differential equation of interfacial shear stresses of RC beams strengthened with FRP plate is established. And the calculated formula of interfacial shear stresses is deduced under mid-span concentrated loads.

\section{Analytical Model}

\section{Basic Assumptions}

A differential section $d x$, can be cut out from the FRP strengthened RC beams. The concrete beam is made from three materials: RC concrete, adhesive layer and FRP plate. According to the characteristics and mechanical behavior of FRP strengthened RC beams, we make the following assumptions for simplicity of the problems before starting the derivations:

1. Adherends are homogeneous and linear elastic;

2. Deformations of FRP plate and concrete are due to bending moments, axial and shear forces; 
3. The adhesive layer is assumed to be subject to stresses invariant across its thickness;

4. The curvatures of FRP plate and concrete beam are assumed to be the same.

\section{Governing Differential Equation}

Considering a typical infinitesimal unit body of the FRP strengthened RC beams as shown in Fig.1.

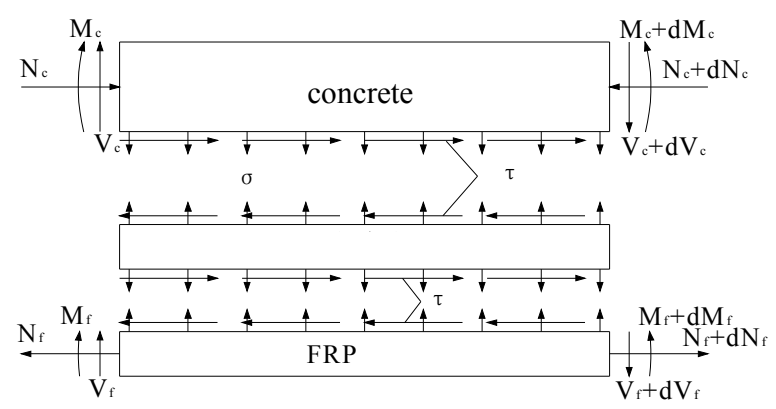

Fig.1 The force graph of unit body

Ignore the axial force, bending moment and shear force of adhesive layer, so the adhesive layer is exposed only to the interfacial shear and normal stresses. Adhesive layer is mainly transmitted shear force and shear deformation. $u(x, y)$ and $v(x, y)$ are the horizontal and vertical displacements respectively at any point in the adhesive layer as defined in Fig.1. The interfacial shear stresses are denoted by $\tau(x)$. The corresponding shear stress is given as follows.

$$
\tau(x)=G_{a}\left(\frac{d u(x, y)}{d y}+\frac{d v(x, y)}{d x}\right)
$$

Where $G_{a}$ is the shear modulus of the adhesive layer.

According to the basic assumptions, Eq. 2 can be rewritten as follow.

$$
\frac{d u(x, y)}{d y}=\frac{1}{t_{a}}\left(u_{f}(x)-u_{c}(x)\right)
$$

Where $u_{c}(x)$ and $u_{f}(x)$ are the longitudinal displacements at the base of concrete beam and the top of FRP plate respectively, and $t_{a}$ is the thickness of the adhesive layer.

Substitute Eq. 2 into Eq.1. Eq. 3 can be rewritten as follow.

$$
\frac{d \tau(x)}{d x}=G_{a}\left[\frac{1}{t_{a}}\left(\frac{d u_{f}(x)}{d x}-\frac{d u_{c}(x)}{d x}\right)+\frac{d^{2} v(x, y)}{d x^{2}}\right]
$$

The formula of $\frac{d^{2} v(x, y)}{d x^{2}}$ in Eq.3 is very small and thus is ignored in the following derivation. Eq. 4 can be rewritten as follow.

$$
\frac{d \tau(x)}{d x}=\frac{G_{a}}{t_{a}}\left(\varepsilon_{f}(x)-\varepsilon_{c}(x)\right)
$$

Where $\varepsilon_{f}(x), \varepsilon_{c}(x)$ are the strain at the bottom of concrete beam and the top of FRP plate respectively.

Considering axial and bending deformations, the formulas are given as below.

$$
\varepsilon_{c}(x)=\frac{M_{c}(x) y_{1}}{E_{c} I_{c}}-\frac{N_{c}(x)}{E_{c} A_{c}}
$$




$$
\varepsilon_{f}(x)=-\frac{M_{f}(x) y_{2}}{E_{f} I_{f}}+\frac{N_{f}(x)}{E_{f} A_{f}}
$$

Where $t_{f}, b_{f}$ are the thickness and width of the FRP plate respectively; $E_{c}, E_{f}, I_{c}, I_{f}$ are elastic modulus, inertia moments of RC beam and FRP plate respectively; $A_{c}, A_{f}$ are cross-sectional areas of RC beam and FRP plate respectively; $M_{c}(x), M_{f}(x), N_{c}(x), N_{f}(x), V_{c}(x), V_{f}(x)$ are the bending moment, axial and shear forces in each adherend while $y_{1}$ and $y_{2}$ are the distances from the bottom of RC beam and the top of FRP plate to their respective centroid. The following equilibrium equations are established as below.

$$
\begin{aligned}
& \frac{d M_{f}(x)}{d x}=V_{f}-\tau b_{f} y_{2} \\
& \frac{d M_{c}(x)}{d x}=V_{c}-\tau b_{f} y_{1} \\
& \frac{d N_{c}(x)}{d x}=\frac{d N_{f}(x)}{d x}=\tau b_{f}
\end{aligned}
$$

Moment equilibrium of the differential segment of the beam in Fig.1 gives

$$
M(x)=M_{f}(x)+M_{c}(x)+N(x)\left(y_{1}+y_{2}+t_{a}\right)
$$

Assuming equal curvature in the concrete beam and FRP plate, the relationship between the moments in the two adherends can be expressed as below.

$$
\frac{M_{f}(x)}{E_{f} I_{f}}=\frac{M_{c}(x)}{E_{c} I_{c}}
$$

Substituting Eq. 5-11 into Eq.4, and get derivative, then the differential equation refers with Eq.12.

$$
\frac{d^{2} \tau(x)}{d x^{2}}=\alpha^{2} \tau-\beta V(x)
$$

Where $\quad \alpha^{2}=\frac{G_{a}}{t_{a}} b_{f}\left[\frac{1}{E_{f} A_{f}}+\frac{1}{E_{c} A_{c}}+\frac{\left(y_{1}+y_{2}\right)^{2}}{E I}\right], \quad \beta=\frac{G_{a}\left(y_{1}+y_{2}\right)}{E I t_{a}} \quad, \quad E I=E_{f} I_{f}+E_{c} I_{c} \quad$ and $V(x)=V_{f}+V_{c}$.

\section{Calculation formulas}

The calculating diagram under mid-span concentrated loads is shown in Fig.2.

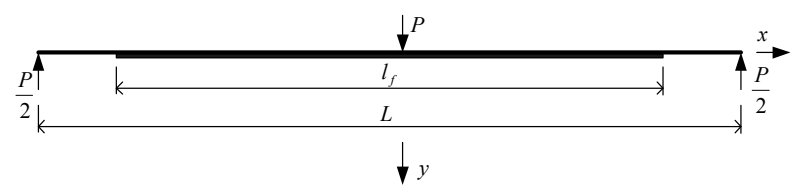

Fig.2. Mid-span concentrated loads

Under mid-span concentrated loads $\mathrm{P}$ (beam length is $L$, length of FRP plate is $l_{f}$ ), there is $V=-\frac{P}{2} \quad\left(0 \leq x \leq \frac{l_{f}}{2}\right)$.Then the solution to differential equation of interfacial shear stresses refers 
with Eq.13 and can be obtained by using the following boundary conditions.

$$
\tau(x)=A_{1} e^{\alpha x}+A_{2} e^{-\alpha x}-\frac{\beta P}{2 \alpha^{2}}
$$

When $x=0$, there is $\tau=0$ and when $x=0.5 l_{f}$, there is $\frac{d \tau}{d x}=-\frac{G_{a} P\left(L-l_{f}\right)\left(y_{1}+y_{2}\right)}{4 E I t_{a}}=-\tau_{b}$. The calculated formula of interfacial shear stresses under mid-span concentrated loads refers with Eq.14.

$$
\tau(x)=\frac{\beta P \alpha e^{-\frac{\alpha}{2} l_{f}}-2 \tau_{b} \alpha^{2}}{2 \alpha^{3}\left(e^{\frac{\alpha}{2} l_{f}}+e^{-\frac{\alpha}{2} l_{f}}\right)} e^{\alpha x}+\frac{\beta P \alpha e^{\frac{\alpha}{2} l_{f}}+2 \tau_{b} \alpha^{2}}{2 \alpha^{3}\left(e^{\frac{\alpha}{2} l_{f}}+e^{-\frac{\alpha}{2} l_{f}}\right)} e^{-\alpha x}-\frac{\beta P}{2 \alpha^{2}}
$$

\section{Conclusions}

Based on the analytical model, the differential equation of interfacial shear stresses of RC beams strengthened with FRP plate is established. And the calculated formula of interfacial shear stresses is deduced under mid-span concentrated loads. The calculation formula of interfacial shear stresses is rational in theory, but the applicability of the calculation formula needs more experiments to verify.

\section{References}

[1] Lianguang Wang. Prestressed Composite Structures of Steel and Concrete. Beijing Science Press (2009) in press. (In Chinese)

[2] A.S. Bouchikhi, A. Lousdad, A. Megueni. On the reduce of interfacial shear stresses in fiber reinforced polymer plate retrofitted concrete beams. Materials and Design, 31 (2010) 1508-1515

[3] Teng J. G, Yuan H. FRP-to concrete interface between two adjacent cracks: Theoretical model for debonding failure. International Journal of Solids and Structures, Vol. 43(2006), p. 5750.

[4] Benachour A., Benyoucef S. Interfacial stress analysis of steel beams reinforced with bonded prestressed FRP plate. Engineering Structures, Vol. 30(2008), p. 3305.

[5] A. Lousdad, A. Megueni, A.S. Bouchikhi. Geometric edge shape based optimization for interfacial shear stress reduction in fiber reinforced polymer plate retrofitted concrete beams. Computational Materials Science, 47 (2010): 911-918

[6] Tim S., John C. Elastic analysis of adhesion stress for the design of a strengthening plate bonded to a beam. Construction and Building Materials, Vol. 20(2006), p. 34.

[7] Fangliang Chen, Pizhong Qiao. Debonding analysis of FRP-concrete interface between two balanced adjacent flexural cracks in plated beams. International Journal of Solids and Structures, Vol. 46 (2009), p. 2618-2628.

[8] S.T. Smith, J.G. Teng. Interfacial stresses in plated beams. Engineering Structures 23 (2001) 857-871.

[9] Zhang Jiwen, Lu Zhitao, Teng Jinguang, Smith S. T. Analysis of the interfacial stresses of the strengthened RC beams externally bonded with CFRP sheets or steel plates [J]. Industrial Construction, 2001, 31(6): 1-4. (In Chinese) 